\title{
Dimensionamento de ampliação do aeroporto de Marabá com base em estudo de previsão de demanda aeroportuária
}

[Capacity expansion goal-setting for the Marabá airport based on a study on airport demand]

\author{
Renan Rios Diniz*
}

Instituto Tecnológico de Aeronáutica (ITA), Brazil

Submitted 7 Nov 2011; received in revised form 12 Dec 2011; accepted 26 Jan 2012

\begin{abstract}
Resumo
Este trabalho investiga a adequação de um projeto de ampliação da capacidade nominal do aeroporto de Marabá para 500.000 passageiros/ano. Para isto se serve de uma abordagem de projeção de demanda por meio de regressões lineares. São analisados econometricamente três modelos, e opta-se por um compromisso entre características de explicação de evolução histórica e capacidade preditiva. Conclui-se que há fortes indicações de que a reforma proposta é insuficiente em escopo.
\end{abstract}

Palavras-Chave: demanda aeroportuária; marabá; aeroportos regionais; investimentos; econometria.

\begin{abstract}
This paper investigates whether a proposed project for increasing the nominal capacity for the Marabá International Airport to 500.000 passengers/year would be adequate. In order to do this it uses a demand forecast approach through use of linear regressions. Three models are econometrically analyzed, and an option is made for a compromise between explanation of historical evolution and predictive capacity characteristics. It is concluded that strong indications are present that the proposed reform is insufficient in its scope.
\end{abstract}

Key words: airport demand; marabá; regional airports; investments; econometrics.

*Email: rios.diniz@gmail.com.

\section{Recommended Citation}

Diniz, R. R. (2013) Dimensionamento de ampliação do aeroporto de Marabá com base em estudo de previsão de demanda aeroportuária. Journal of Transport Literature, vol. 7, n. 1, pp. 147-162.

- JTL/RELIT is a fully electronic, peer-reviewed, open access, international journal focused on emerging transport markets and published by BPTS - Brazilian Transport Planning Society. Website www.transport-literature.org. ISSN 2238-1031.

This paper is downloadable at www.transport-literature.org/open-access. 


\section{Introdução}

O município de Marabá, no estado do Pará, tem apresentado altas taxas de crescimento econômico e demográfico. Este crescimento, muito acima da média nacional, agrava os desafios de acessibilidade inerentes a um município amazônico.

O Aeroporto de Marabá (João Correa da Rocha) endereça parte deste desafio. Este aeroporto tem ampliado sua oferta de vôos e permite boa conectividade nacional, mas mostra sinais de saturação de sua capacidade.

Tendo isto em vista, foi noticiada em 2011 a possibilidade um projeto de ampliação do aeroporto que levaria a capacidade deste a 500.000 passageiros/ano. Este projeto, ainda não licitado, não é de execução certa, mas dado o investimento necessário e o rápido crescimento da cidade é pertinente uma discussão sobre que projeto seria adequado para o município. Para tal discussão é essencial que seja bem-modelada a demanda do aeroporto de Marabá.

Este estudo modela a demanda para o Aeroporto de Marabá por meio de uma metodologia de regressão linear. Entre três modelos, um é escolhido para uma projeção de demanda segundo três cenários. A partir dessa projeção conclui-se que há grande probabilidade de a reforma proposta ser insuficiente.

Este trabalho está organizado da seguinte forma: na seção 2 é feita uma caracterização do Município de Marabá e de seu aeroporto. Na seção 3 são caracterizados os dados utilizados, e é feita uma análise preliminar. Na seção 4 é feita uma descrição da metologia de regressão linear. Na seção 5 são definidos e calculados os modelos de regressão linear. Na seção 6 é realizada a projeção de demanda. A seção 7 explicita as limitações do modelo e, à luz delas, analisa a adequação do projeto de ampliação proposto. 


\section{Característcas do Aeroporto e do Município de Marabá}

\section{Caracterização do Município de Marabá}

Marabá tem se destacado pela velocidade de seu crescimento. De acordo com o Censo 2010 do IBGE o município possuía 233.669 habitantes, ao passo que o Censo 2000 havia identificado 196.468 habitantes e o censo 1991, 123.688. Isso representa, para os dois intervalos, taxas de crescimento anualizadas de 3,46\% e 3,35\%, respectivamente. Estes valores são bem superiores aos correspondentes nacionais, $1,18 \%$ e 1,60\%, respectivamente.

Quanto ao PIB, o descolamento em relação à média nacional é ainda maior, como mostra a Tabela 1. Este crescimento é motivado pela mineração na região vizinha de Carajás e pela indústria siderúrgica a ela associada.

Tabela 1- Evolução do PIB de Marabá e nacional

\begin{tabular}{|c|c|c|c|}
\hline Ano & PIB Marabá & $\begin{array}{c}\text { Crescimento } \\
\text { Marabá }\end{array}$ & $\begin{array}{c}\text { Crescimento } \\
\text { Brasil }\end{array}$ \\
\hline 2008 & $\mathrm{R} \$ 3.593 .892 .000,00$ & $15,4 \%$ & $5,20 \%$ \\
\hline 2007 & $\mathrm{R} \$ 3.115 .485 .000,00$ & $18,8 \%$ & $6,10 \%$ \\
\hline 2006 & $\mathrm{R} \$ 2.621 .407 .000,00$ & $26,0 \%$ & $4,00 \%$ \\
\hline 2005 & $\mathrm{R} \$ 2.079 .838 .000,00$ & $41,4 \%$ & $3,20 \%$ \\
\hline 2004 & $\mathrm{R} \$ 1.470 .758 .000,00$ & $54,3 \%$ & $5,70 \%$ \\
\hline 2003 & $\mathrm{R} \$$ 953.425.000,00 & $\mathrm{N} / \mathrm{A}$ & $1,10 \%$ \\
\hline Fonte: IBGE (2011) & \\
\hline
\end{tabular}

Em adição ao acesso aeroviário, Marabá conta com três saídas rodoviárias: a BR-222, que dá acesso a Belém e aos estados do nordeste, a BR-230, Transamazônica, que vai da Paraíba ao oeste da Amazônia, mas com vários trechos em condições péssimas de trânsito, e a PA-150, que permite o escoamento de bens para o porto de Vila do Conde, em Barbacena, próximo a Belém.

\section{Caracterização do Município de Marabá}

O aeroporto de Marabá, de acordo com a Infraero, movimentou em 2010242.415 passageiros, e 1.725,354 T em carga. 
A infraestrutura principal consiste em uma pista de pouso de 2000 x $45 \mathrm{~m}$, três posições de pátio e um terminal de passageiros de $603 \mathrm{~m} 2$. Esta infraestrutura não condiz com o tráfego do aeroporto.

De acordo com a ANAC (2011) a cidade possui ligações diretas e regulares com Belém, Brasília, Belo Horizonte, Altamira, Carajás, Paraupebas, Ourilândia do Norte, Araguaína e Redenção, conforme ilustrado pela Figura 1.

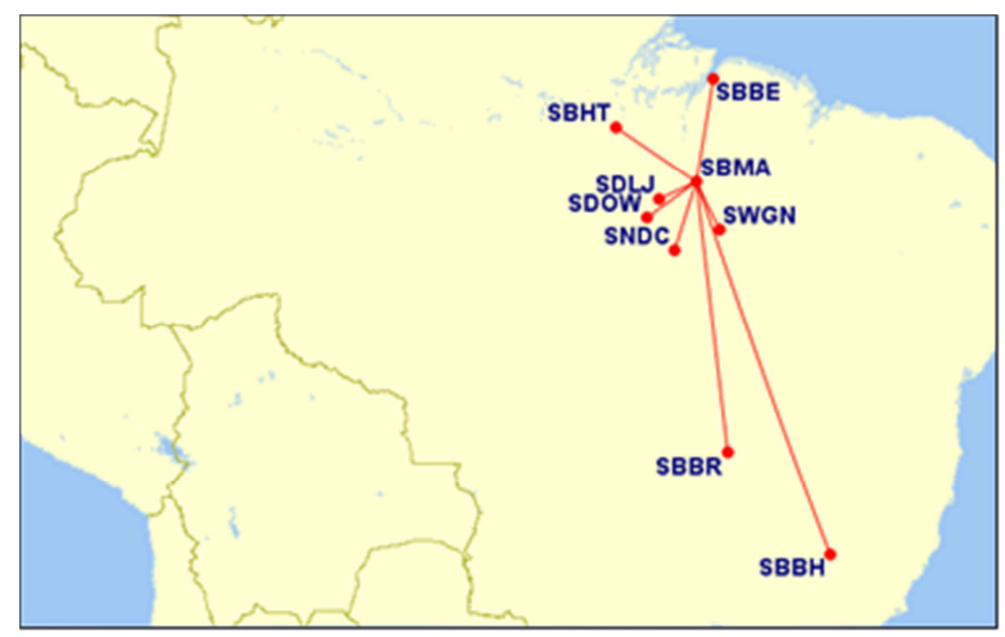

Figura 1 - Rotas regulares do aeroporto de Marabá

São quatro as companhias que operam rotas regulares em Marabá: TAM, Gol, TRIP e Sete. A operação da TRIP é recente, tendo se iniciado em Agosto de 2011. A Gol iniciou suas operações em Março de 2007.

\section{Descrição e análise inicial da base de dados utilizada}

A série utilizada consistiu em um bando de dados, proveniente da INFRAERO, contendo dados mensais de passageiros transportados no período de Janeiro de 1990 a Dezembro de 2010. O tráfego de passageiros é divido nas categorias:

- Doméstico local (paxdom_loc) - tráfego doméstico de passageiros com origem ou destino no aeroporto

- Doméstico de conexão (paxdom_con) - tráfego doméstico de passageiros que realizam conexões no aeroporto 
- Doméstico on-board (paxdom_bor) - tráfego doméstico de passageiros que realizam escala, sem desembarque, no aeroporto

- Doméstico total (paxdom) - tráfego doméstico local e de conexões

- Internacional local (paxdom_loc) - tráfego internacional de passageiros com origem ou destino no aeroporto

- Internacional de conexão (paxdom_con) - tráfego internacional de passageiros que realizam conexões no aeroporto

- Internacional on-board (paxdom_bor) - tráfego internacional de passageiros que realizam escala, sem desembarque, no aeroporto

- Internacional total (paxdom) - tráfego internacional local e de conexões

Neste estudo, dado que Marabá não possui tráfego aéreo internacional, não foram utilizdas as categorias internacionais.

Adionalmente, no intervalo de Janeiro de 1997 a Dezembro de 2010 a série possui valores mensais para o PIB brasileiro (pib), deflacionados pelo IPCA, para o yield doméstico (yield_dom), e para o yield internacional (yield_int), entre as principais.

O Gráfico 1 mostra a série de tráfego total de passageiros (paxdom) entre 1900 e 2010.

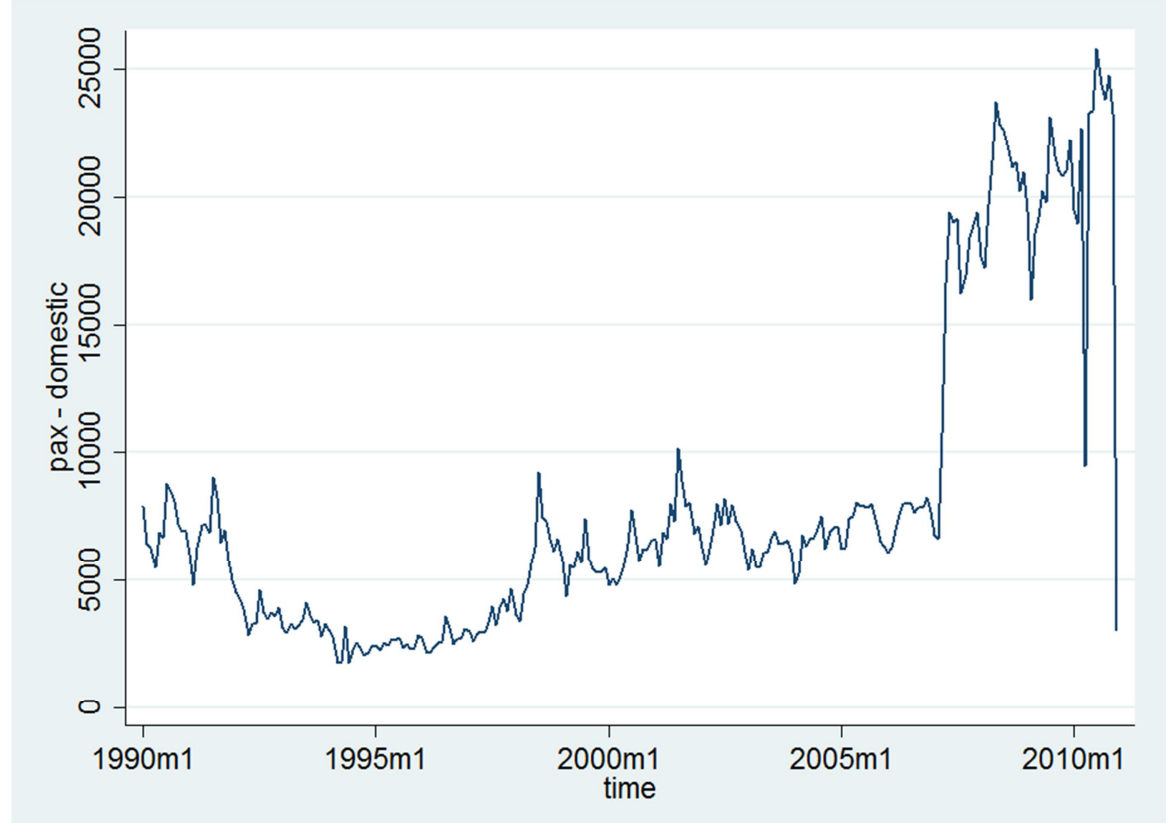

Gráfico 1 - Tráfego de passageiros em Marabá 
Observa-se que há uma quebra de tendência com crescimento abrupto no início de 2007, período de entrada da Gol.

O Gráfico 2 mostra gráficos de dispersão entre o tráfego de passageiros e as séries de PIB e yield nacionais. Embora seja possível ver a correlação entre passageiro e PIB é mais difícil ver a relação entre passageiros e yield nacional. Isto sugere a hipótese de que um fator local tenha mais influência que o yield na formação da demanda.

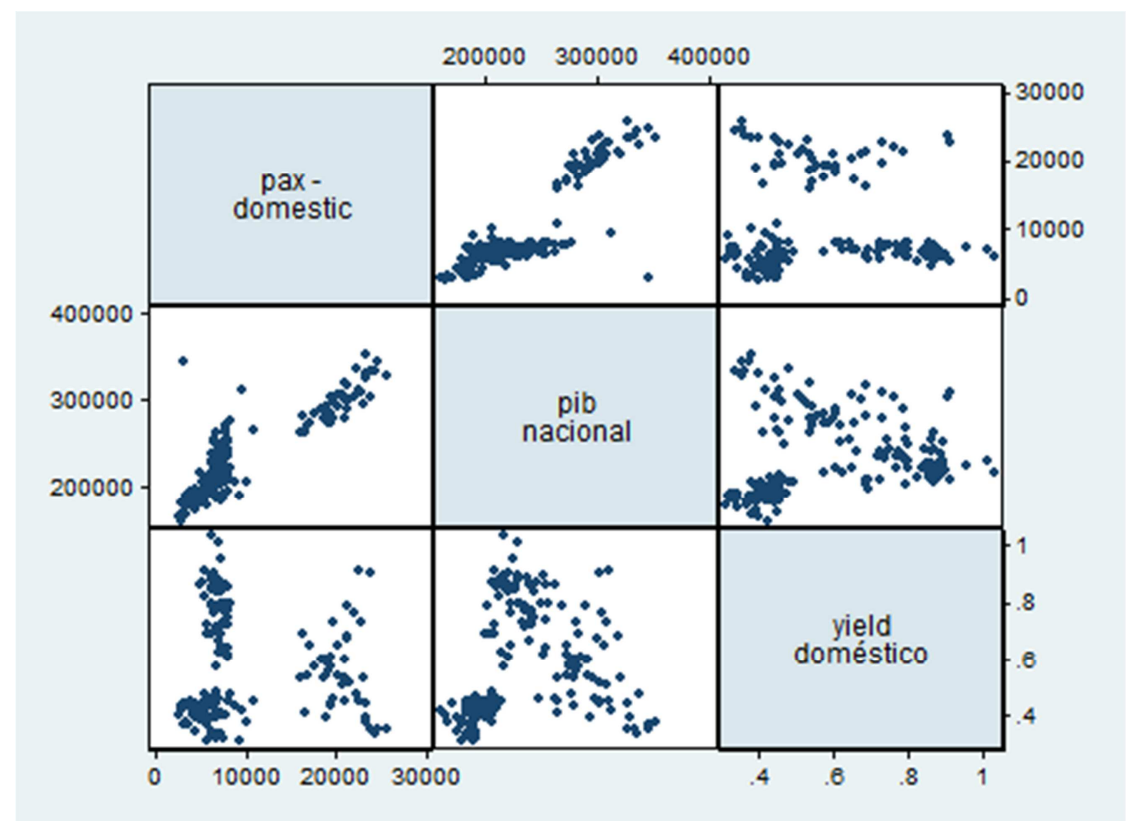

Gráfico 2 - Dispersões entre passageiros, PIB nacional e yield doméstico

\section{Metodologia de regressão linear}

A projeção da demanda de Marabá foi realizada por meio de uma abordagem de regressão linear. Rocha (2010) representa um precedente de uso desta metodologia para ao caso de aeroportos regionais.

Dessa forma assume-se que o tráfego pode ser expresso pela equação (1):

$$
\text { Paxdom }=\beta_{0}+\beta_{1} x_{1}+\beta_{2} x_{2}+\ldots+\beta_{n} x_{n}+u
$$

$\mathrm{Na}$ equação os $\mathrm{x}_{\mathrm{i}}$ são variáveis que determinam a demanda, $\beta_{\mathrm{i}}$ coeficientes destas variáveis e $\mathrm{u}$ um fator associado a influências não identificadas na demanda. 
Dessa forma o a demanda para qualquer tempo é estimada pela equação (2):

$$
\text { Paxdom }=\widehat{\beta}_{0}+\widehat{\beta}_{1} \mathrm{x}_{1}+\widehat{\beta}_{2} \mathrm{x}_{2}+\ldots+\widehat{\beta}_{\mathrm{n}} \mathrm{x}_{\mathrm{n}}
$$

Este modelo assume que os desvios-padrões dos coeficientes sejam constantes ao longo da série. Tendo em mente a possibilidade de variação dos desvios-padrões foi adotada uma correção de heterocedasticidade. Foi também realizado um ajuste de autocorrelação.

Foram diversas as modelagens adotadas para a regressão. Neste trabalho, tendo em vista o questionamento inicial e a geração de conhecimento, serão abordadas as três principais.

\section{Cálculo dos modelos de regressão linear}

\section{Modelo 1: Análise PIB x Yield}

Como ponto de partida para investigação foi assumido que a demanda seria definida por:

- $\quad$ PIB brasileiro (PIB)

- Yield (yielddom)

Isto equivale a considerar a demanda na forma da equação 3 :

$$
\text { Paxdom }=\widehat{\beta}_{0}+\widehat{\beta}_{1} \text { pib }+\widehat{\beta}_{2} \text { yielddom }
$$

Dessa forma foram encontrados os resultados da Tabela 2. 
Tabela 2 - Resultados de primeira tentativa de regressão linear

\begin{tabular}{|c|c|c|c|c|c|c|c|}
\hline \multicolumn{8}{|c|}{$\begin{array}{l}\text { Estimates efficient for homoskedasticity only } \\
\text { statistics robust to heteroskedasticity and autocorrelation } \\
\text { kernel=Bartlett; bandwidth= } \\
\text { time variable }(t): \text { time }\end{array}$} \\
\hline \multicolumn{2}{|c|}{$\begin{array}{l}\text { Total (centered) SS } \\
\text { Total (uncentered) SS } \\
\text { Residual SS }\end{array}$} & \multicolumn{3}{|c|}{$\begin{array}{l}=7098805863 \\
=2.38955 e+10 \\
=1558689601\end{array}$} & \multirow{2}{*}{\multicolumn{2}{|c|}{$\begin{aligned} \text { Number of obs } & = \\
\mathrm{F}(2,165) & = \\
\text { Prob }>\mathrm{F} & = \\
\text { Centered R2 } & = \\
\text { Uncentered R2 } & = \\
\text { Root MSE } & = \\
\mathrm{P}>|\mathrm{t}| \quad[95 \% \text { conf. } & \end{aligned}$}} & \multirow{2}{*}{$\begin{array}{rr}= & 168 \\
= & 94.04 \\
= & 0.0000 \\
= & 0.7804 \\
= & 0.9348 \\
= & 3074 \\
& \\
& \end{array}$} \\
\hline paxdom & Coe & & $\begin{array}{l}\text { Robust } \\
\text { std. Err. }\end{array}$ & $t$ & & & \\
\hline $\begin{array}{r}\text { pib } \\
\text { yielddom } \\
\text { _cons }\end{array}$ & $\begin{array}{l}.12609 \\
-5723.6 \\
-16493 .\end{array}$ & $\begin{array}{l}929 \\
685 \\
.84\end{array}$ & $\begin{array}{l}.0095295 \\
1542.966 \\
1734.881\end{array}$ & $\begin{array}{l}13.23 \\
-3.71 \\
-9.51\end{array}$ & $\begin{array}{l}0.000 \\
0.000 \\
0.000\end{array}$ & $\begin{array}{r}.1072774 \\
-8770.186 \\
-19919.27\end{array}$ & $\begin{array}{r}.1449084 \\
-2677.183 \\
-13068.41\end{array}$ \\
\hline \multicolumn{8}{|c|}{ Included instruments: pib yielddom } \\
\hline \multicolumn{8}{|c|}{$\begin{array}{r}(1) \\
\text { paxdom }\end{array}$} \\
\hline \multicolumn{8}{|c|}{\begin{tabular}{|ll} 
pib & $2.9859 \star \star \star$ \\
yielddom & {$[0.170]$} \\
& {$[0.3364 \star \star \star$} \\
& {$[0.089]$}
\end{tabular}} \\
\hline \multicolumn{8}{|l|}{$\begin{array}{ll}N \\
\text { adj. }\end{array}$} \\
\hline \multicolumn{8}{|c|}{$\begin{array}{l}\text { Notas: } \\
\text { - Estimativas de elasticidades apresentadas na média amostral } \\
\text { - Erros padrões estimados em colchetes } \\
\text { - Representações de p-valor: *** } p<0.01 \text {, ** } p<0.05 \text {, * } p<0.10\end{array}$} \\
\hline
\end{tabular}

$\mathrm{O}$ valor de $\mathrm{R}^{2}$ ajustado para o modelo é 0,778 . Os valores dos coeficientes do PIB e do yield apresentam confiança superior a $99 \%$.

O mapeamento gráfico da função de regressão resultante sobre os pontos de dados utilizados gera o Gráfico 3: 


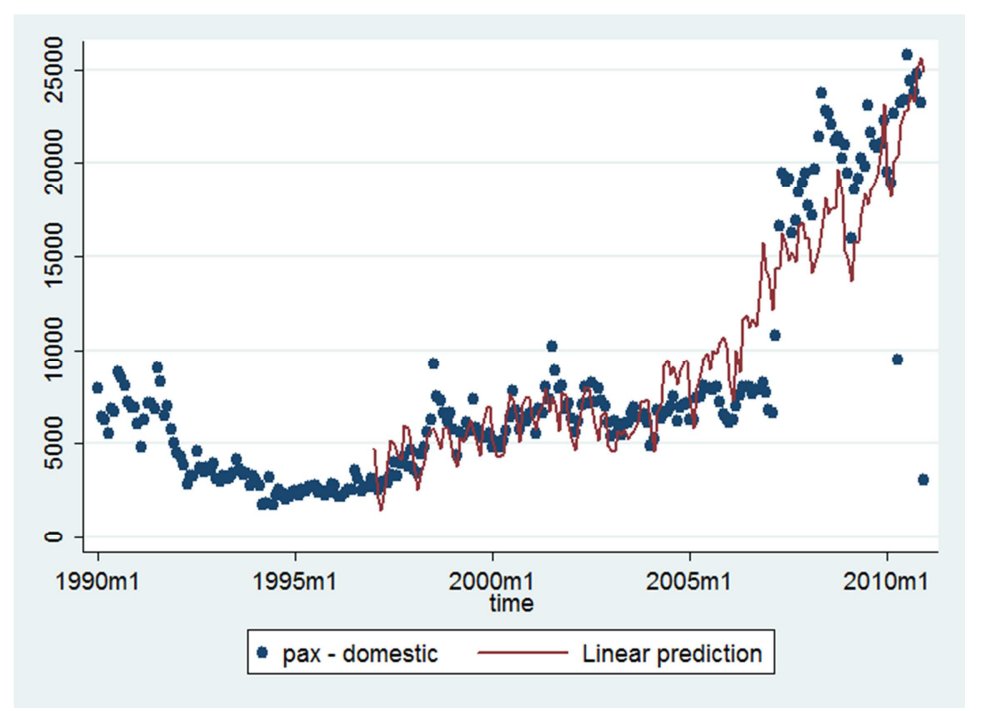

Gráfico 3 - Função de regressão com base em PIB e Yield

Observa-se que a função, embora apresente bom encaixe no início e no final da série, apresenta grandes desvios próximos à entrada da Gol, o que justifica o próximo modelo.

\section{Modelo 2: Análise PIB x Yield com exclusão da transição}

Neste modelo é incluída uma variável dummy de valor 1 após a entrada da Gol e 0 antes. Dessa forma a função de regressão passa a ser dada pela equação (4):

$$
\text { Paxdom }=\widehat{\beta}_{0}+\widehat{\beta}_{1} \text { pib }+\widehat{\beta}_{2} \text { yielddom }+\widehat{\beta}_{3} \text { dgol }
$$

Realizados os cálculos são obtidos os resultados da Tabela 3.

$\mathrm{O}$ valor de $\mathrm{R}^{2}$ ajustado para o modelo é bastante superior, 0,881 . Os valores dos coeficientes do PIB e da dummy de entrada da Gol apresentam confiança superior a 99\%. O coeficente do yield entretanto, passa a ter p-valor 0,969, e portanto perde a significância estatística. Além disso seu valor é quase nulo, o que de forma contra-intuitiva, indica que neste modelo a demanda não sofre influência dos preços (por distância) de passagens. 
Tabela 3 - Resultados de segunda tentativa de regressão

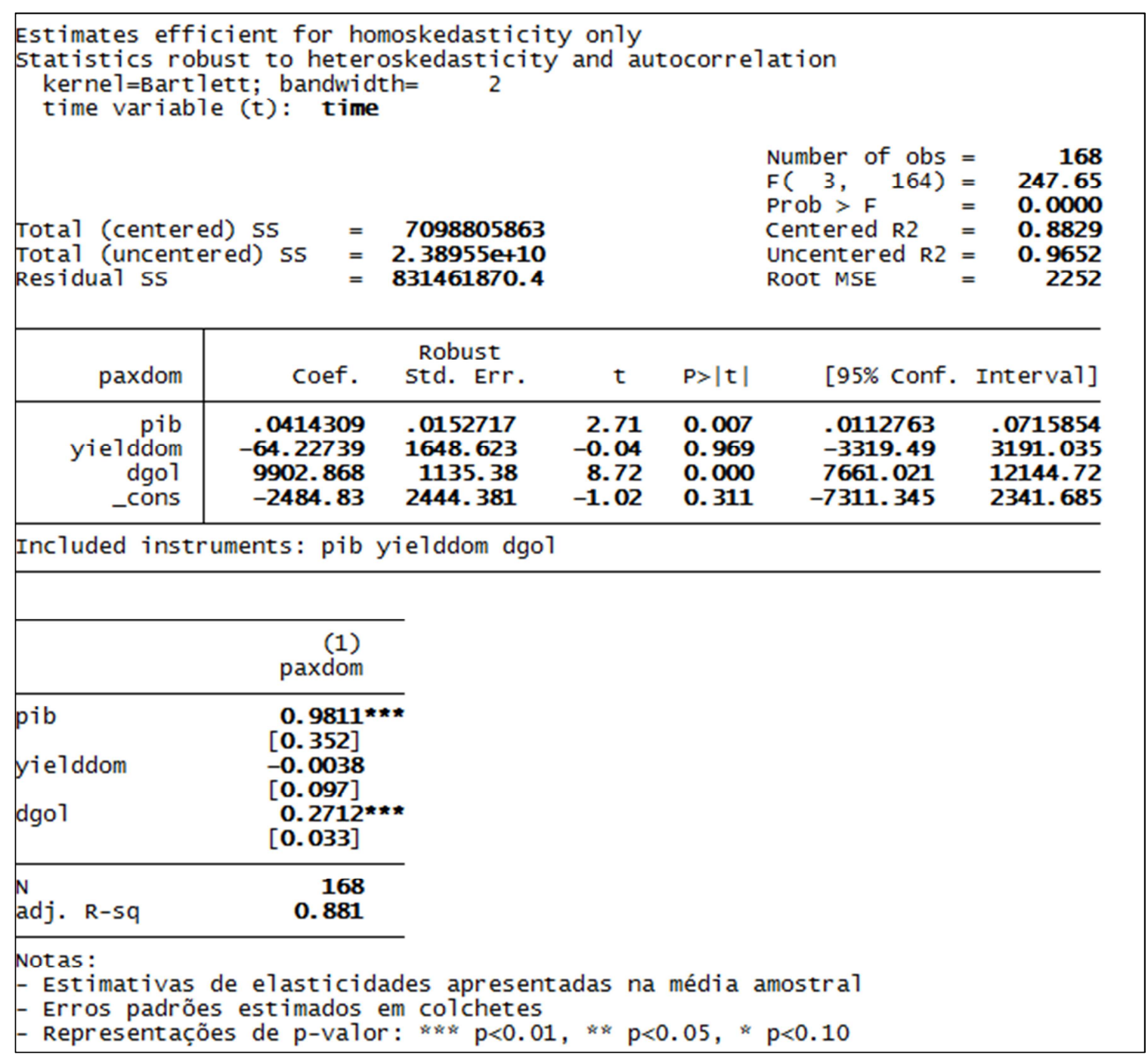

Isto gera complicações para a modelagem da demanda com esta configuração. Reforçando isso, observa-se no Gráfico 4 que a demanda é subestimada após a entrada da Gol. 


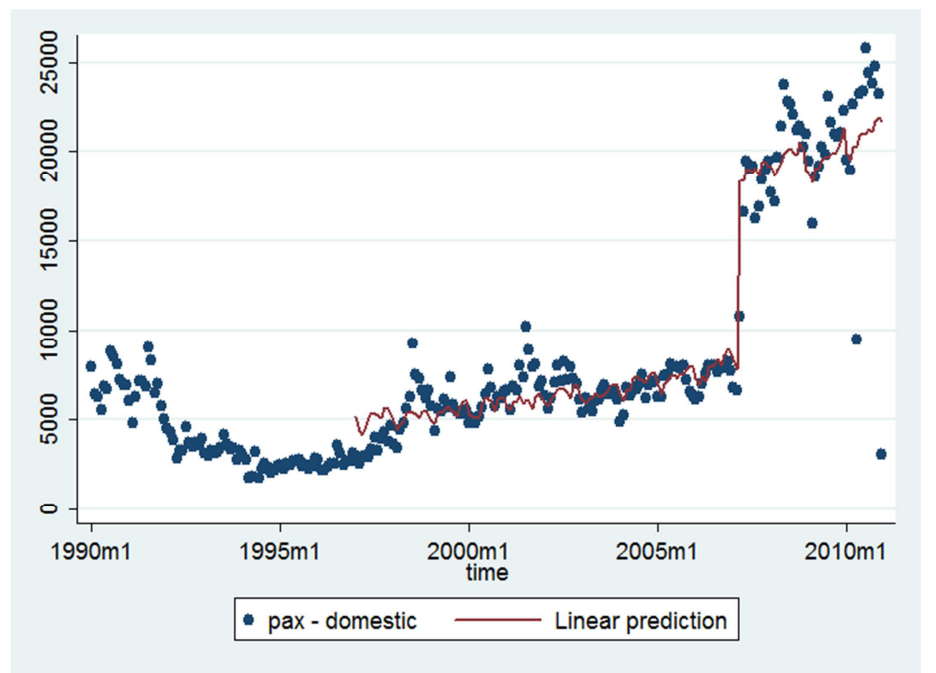

Gráfico 4 - Função de regressão com base em PIB, Yield e atuação da Gol

Este comportamento anômalo deriva do fato de maior parte da variação da demanda no caso de Marabá ter sido resultante da entrada da Gol, o que ofusca efeitos do yield. Saber a influência do yield, no entanto, é de grande valor para a previsão de demanda futura.

Isto justificou a elaboração de um modelo mais refinado de previsão.

\section{Modelo 3: Análise PIB x Yield x "Efeito Gol"}

A análise do gráfico da primeira regressão (Gráfico 3) sugere que a entrada da Gol tem maior influência sobre a demanda logo após o início dos vôos da Gol, uma vez que o maior desvio se dá próximo ao início da atuação da empresa em Marabá.

Dessa forma, em vez do uso de uma dummy convecional de entrada da Gol foi utilizada uma variável correspondente ao "Efeito Gol” em Marabá (defgol) que possui valor 0 a qualquer tempo exceto no período de Março de 2007 a Julho de 2008.

Dessa forma a função de regressão adotada foi a da equação 5:

$$
\text { Paxdom }=\widehat{\beta}_{0}+\widehat{\beta}_{1} \text { pib }+\widehat{\beta}_{2} \text { yielddom }+\widehat{\beta}_{3} \text { defgol }
$$

Com isso foram obtidos os resultados da Tabela 4: 
Tabela 4 - Resultados do modelo final de regressão

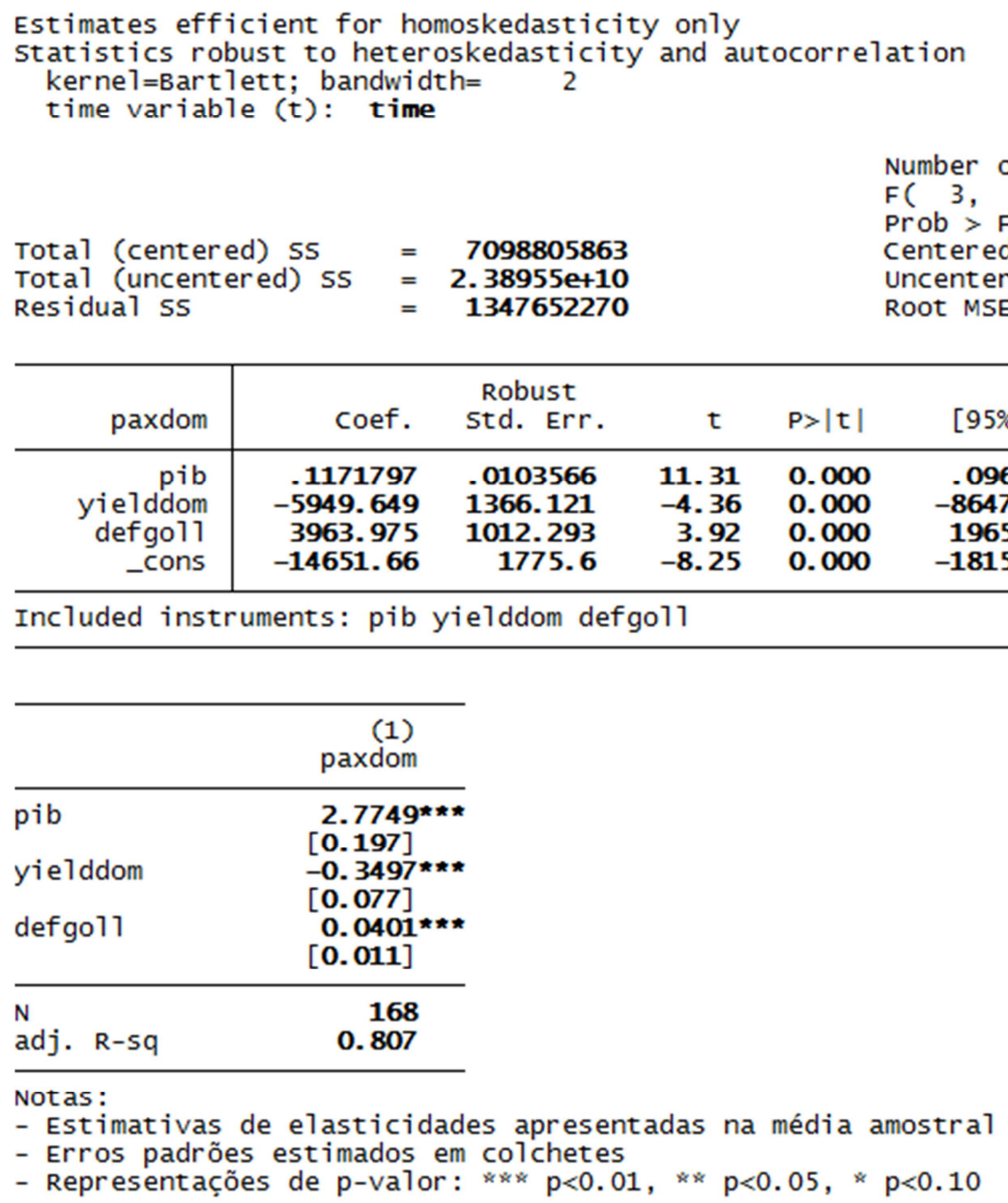

O valor de R2 ajustado para o modelo é intermediário entre os dois modelos. No entanto, os valores dos coeficientes do PIB, da dummy de entrada da Gol e do yield apresentam confiança superior a 99\%, o que facilita o uso deste modelo para projeção.

A análise da função obtida (Gráfico 5) mostra que há uma subestimação do tráfego logo após o final arbitrário da validade da dummy do "Efeito Gol". Esta subestimação, no entanto, se reduz com o avanço da série. Isso pode ser interpretado como resultado de o efeito não ser pontual, mas disperso no tempo e decrescente. A perda em valor de $\mathrm{R}^{2}$, desta forma, pode ser encarada como resultado de um compromisso realizado entre os dois modelos. A meta deste compromisso é o melhor modelo preditivo, e não o melhor ajuste histórico.

Cabe observar, entretanto, que tal abordagem ao não considerar uma variável de entrada da Gol no final da série tende a ser conservadora. 


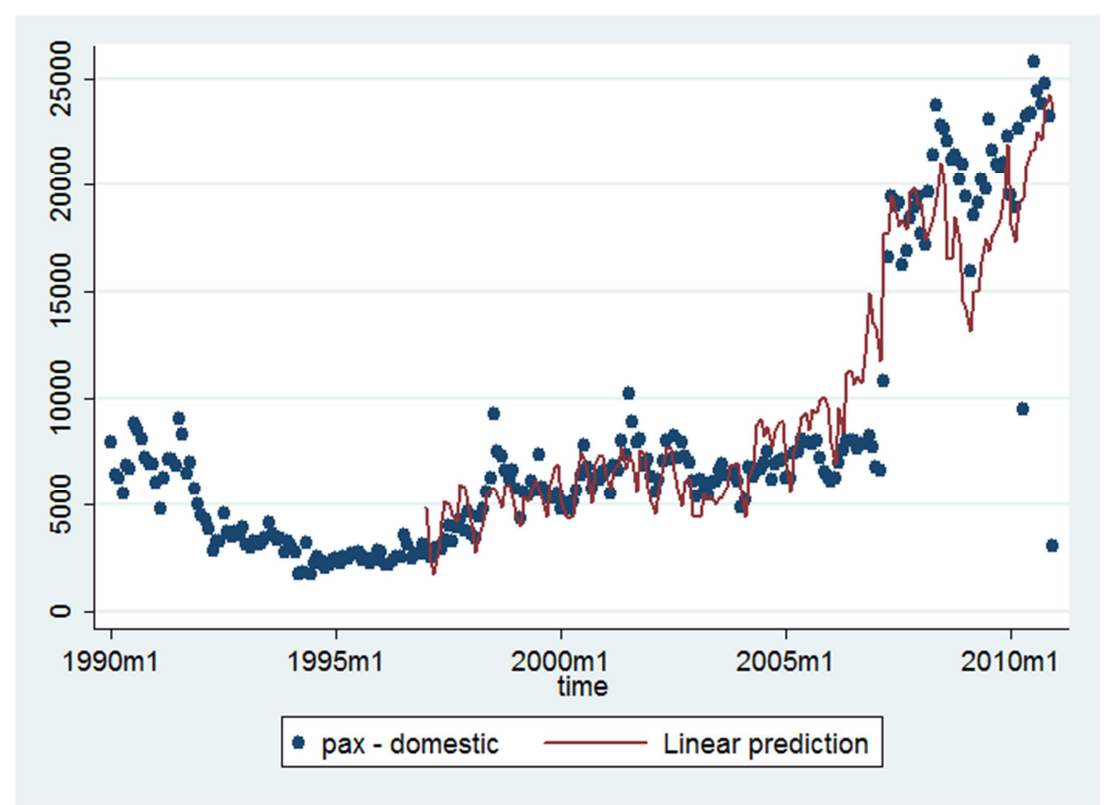

Gráfico 5 - Função de regressão baseada em PIB, Yield e "Efeito Gol"

\section{Previsão de demanda para o Aeroporto de Marabá}

Com a função de regressão definida foi possível projetar a demanda do Aeroporto de Marabá. Para garantir maior acurácia foram utilizados dados de 2011 da INFRAERO referentes aos meses de Janeiro a Outubro. Para a estimativa do tráfego de 2011, utilizado como base de projeção, foi assumido que a proporção de tráfego correspondente ao perídodo de Janeiro a Outubro em relação ao resto do ano seria a mesma de 2010.

O tráfego de passageiros de Janeiro a Outubro de 2011 foi de 264.647 passageiros, o que implicou uma estimativa de 296.850 passageiros para todo o ano de 2011.

Foram definidos três cenários de projeção - Otimista, Base e Pessimista, que variam em suas premissas de evolução do PIB e do yield. De acordo com o Boletim Focus do Banco Central do Brasil de 18 de Novembro de 2011 o crescimento esperado pelo mercado para o PIB de 2011 seria de 3,16\%. Considerando que 2011 tem sido fortemente impactado pelas crises de dívida americana e européia este valor foi adotado como base do cenário pessimista, e os cenários base e otimista foram definidos em níveis superiores.

A Tabela 5 mostra os parâmetros para os três cenários: 
Tabela 5 - Cenários para projeção de demanda

\begin{tabular}{|c|c|c|}
\hline Cenário & PIB & Yield \\
\hline Pessimista & $3,16 \%$ a.a (Focus) & $+10 \%$ do valor de 2010 até 2014 \\
\hline Base & $3,95 \%$ a.a. (1,25 Focus) & $-10 \%$ do valor de 2010 até 2014 \\
\hline Otimista & $4,74 \%$ a.a. (1,5 Focus) & $-20 \%$ do valor de 2010 até 2014 \\
\hline
\end{tabular}

A demanda foi projetada ano a ano até 2014, uma vez que a confiabilidade do modelo é maior no curto prazo. Estes valores, no entanto foram comparados com benchmarks de crescimento, e podem servir de base para a verificação da adequação da capacidade do aeroporto para um horizonte de tempo mais logo.

A Tabela 6 mostra as projeções de demanda para 2014. O Gráfico 6, além destas, mostra a evolução esperada da demanda e a compara com benchmarks de crescimento.

Tabela 6 - Projeções de demanda para 2014

\begin{tabular}{|c|c|c|}
\hline Cenário & Passgeiros-ano & Crescimento anual \\
\hline Pessimista & 367.052 & $7,33 \%$ \\
\hline Base & 408.749 & $11,25 \%$ \\
\hline Otimista & 440.385 & $14,05 \%$ \\
\hline
\end{tabular}

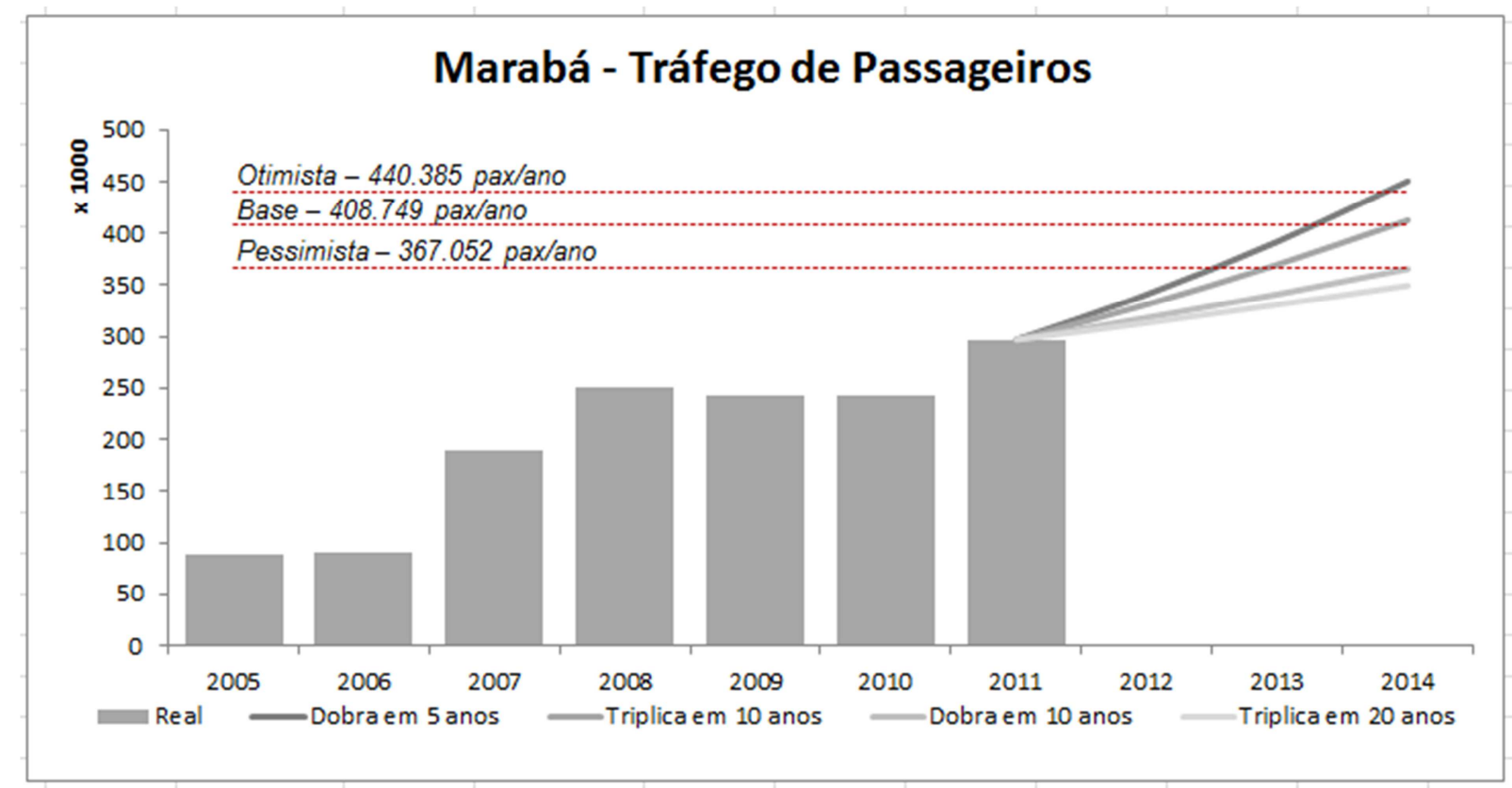

Gráfico 6 - Projeções de demanda para o aeroporto de Marabá 
O cenário base se encaixa em um cenário de triplicação do tráfego em 10 anos. Desta forma em 10 anos o tráfego esperado é da ordem de 890.000 passageiros/ano. Em 5 anos a demanda alcança valores superiores a 500.000 passageiros/ano.

\section{Implicações para projeto de ampliação e limitações}

\section{Limitações do modelo adotado}

O modelo apresenta diversas limitações:

- Não-tolerância a não linearidade nas elasticidades

- Não adaptação a alterações na infraestrutura do aeroporto

- Utilização apenas de variáveis nacionais de PIB e Yield, e não locais

- Incerteza nas projeções de PIB e Yield

\section{Implicações para um projeto de ampliação da capacidade}

A regressão escolhida, como dito anteriormente, é conservadora. Além disso, como o aeroporto opera atualmente com excesso de tráfego, é bastante provável, que apesar das limitações do modelo, a ampliaçao proposta se esgote em um período de tempo muito inferior ao ideal para projeto, uma vez que o cenário base prevê que a capacidade-alvo de 500.000 passageiros-ano seja alcançada em 5 anos.

\section{Conclusões}

Foram desenvolvidos três modelos de regressão linear para modelar a demanda do Aeroporto de Marabá. Um deles mostrou indicações de capacidade preditiva, mas teve relativamente baixo ajuste com a série histórica. Um segundo modelo, considerando a influência da entrada de uma nova companhia, foi capaz de explicar a série histórica, mas, além de fornecer indicações desfavoráveis de poder preditivo, levou a conclusões sem sentido econométrico em relação à influência do yield.

Um terceiro modelo foi um compromisso entre os dois anteriores, e combinou, em parte o encaixe histórico de um modelo e as indicações de capacidade preditiva de outro em uma abordagem justificável econometricamente.

Este modelo projetou uma demanda para o aeroporto que, em um cenário realista, o faria triplicar de tráfego em 10 anos e atingir 500.000 passageiros/ano em 5. Estes números são 
indicações consideráveis da inadequação de um projeto de ampliação que tenha esta capacidade de passageiros como alvo, e justificam a realização de estudos tendo em vista a definição de uma meta mais ambiciosa de ampliação.

\section{Referências}

Alves, C. J. P. and Amaral, F. C. F. (2012) A situação da infraestrutura aeroportuária na região Norte e seu potencial apoio à aviação regional. Journal of Transport Literature, vol. 6, n. 1 .

Amorim, H. C. (2007) A Gol ainda é uma Empresa Low-Fare? Journal of Transport Literature, vol. 1, n. 1 , pp. 23-45.

Bettini, H. F. (2007) Um Retrato da Aviação Regional no Brasil. Journal of Transport Literature, vol. 1, n. 1, pp. 46-65.

Bettini, H. F. A. J. and Oliveira, A. V. M. (2011) Transporte aéreo regional: entre economias de densidade e custos de transação. Journal of Transport Literature, vol. 5, n. 4, pp. 171-187.

Condé, M. (2011) Estudo e previsão de demanda aeroportuária para a cidade do Rio de Janeiro. Journal of Transport Literature, vol. 5, n. 1, pp. 161-183.

Demant, M. A. R. (2011) Infraestrutura aeroportuária e o desenvolvimento do tráfego aéreo regional no Brasil. Journal of Transport Literature, vol. 5, n. 1, pp. 124-160.

Falcão, V. A. (2013) Demanda aeroportuária de Manaus e sua influência para o setor de turismo da região. Journal of Transport Literature, vol. 7, n. 1.

Fraga, R. (2010) Estudo de Caso: Histórico e Análise Evolutiva da Trip Linhas Aéreas. Journal of Transport Literature, vol. 2, n. 2, pp. 66-93.

Fregnani, J. A., Ferreira, N. S. and Griebeler, M. C. (2009) Análise de Custos Logísticos do Transporte Aéreo Regional. Journal of Transport Literature, vol. 3, n. 2, pp. 7-24.

IBGE, Sinopse Preliminar do Censo 2010, 2011, IBGE, Rio de Janeiro, RJ

IBGE, Sinopse Preliminar do Censo 2000, 2001, IBGE, Rio de Janeiro, RJ

IBGE, Censo Demográfico 1991, Resultados preliminares, 1992, IBGE, Rio de Janeiro, RJ

IBGE, Contas Nacionais Trimestrais, 2011

Kuroda,. E. T., Kalfas, A. J. and Eller, R. S. G. (2012) Análise da produtividade no setor aéreo utilizando função Cobb-Douglas: o caso da GOL. Journal of Transport Literature, vol. 6, n. 2.

Rocha, G. C. (2010) Ensaios sobre a Demanda do Transporte Aéreo Regional. Journal of Transport Literature, vol. 4, n. 1, pp. 114-133.

Santos, F. A. B. (2008) Demanda por Transporte Aéreo e seus Desdobramentos. Journal of Transport Literature, vol. 2, n. 2, pp. 94-113.

Turolla, F. A., Lima, M. F. F. and Ohira, T. H. (2011) Políticas públicas para a melhoria da competitividade da aviação regional brasileira. Journal of Transport Literature, vol. 5, n. 4, pp. $188-231$. 